Rotation-vibration interactions in the spectra of polycyclic aromatic hydrocarbons: Quinoline as a test-case species

O. Pirali, Z. Kisiel, M. Goubet, S. Gruet, M. A. Martin-Drumel' , A. Cuisset, F. Hindle, and G. Mouret

Citation: The Journal of Chemical Physics 142, 104310 (2015); doi: 10.1063/1.4913750

View online: http://dx.doi.org/10.1063/1.4913750

View Table of Contents: http://aip.scitation.org/toc/jcp/142/10

Published by the American Institute of Physics

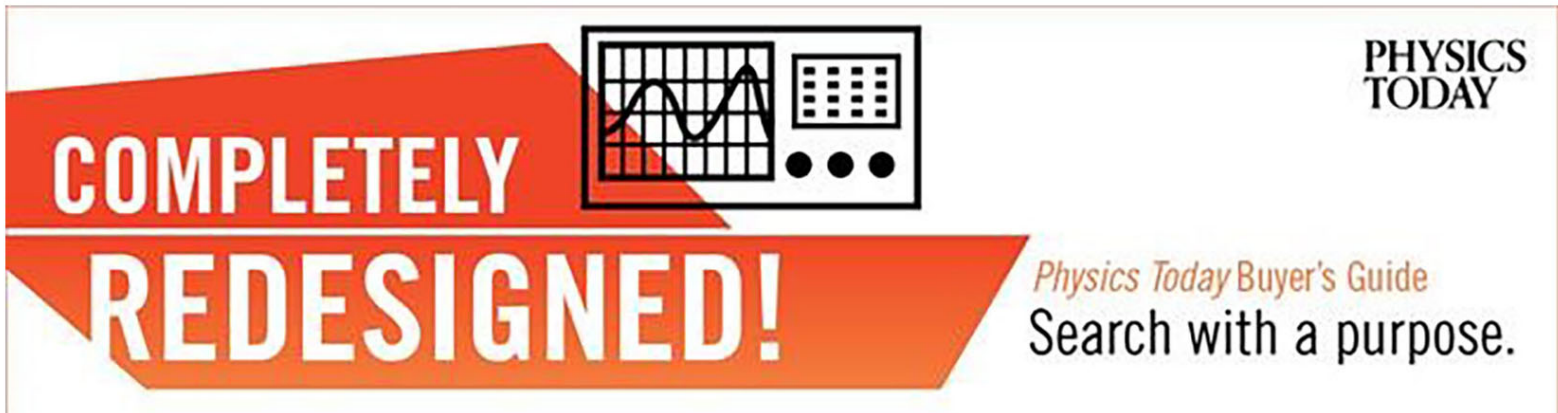




\title{
Rotation-vibration interactions in the spectra of polycyclic aromatic hydrocarbons: Quinoline as a test-case species
}

\author{
O. Pirali, ${ }^{1,2}$ Z. Kisiel, ${ }^{3}$ M. Goubet, ${ }^{4}$ S. Gruet,,${ }^{1,2}$ M. A. Martin-Drumel, ${ }^{5, a)}$ A. Cuisset,${ }^{5}$ \\ F. Hindle, ${ }^{5}$ and G. Mouret ${ }^{5}$ \\ ${ }^{1}$ AILES Beamline, Synchrotron SOLEIL, l'Orme des Merisiers, Saint-Aubin, 91192 Gif-sur-Yvette cedex, France \\ ${ }^{2}$ Institut des Sciences Moléculaires d'Orsay, UMR8214 CNRS - Université Paris-Sud, Bât. 210, \\ 91405 Orsay cedex, France \\ ${ }^{3}$ Institute of Physics, Polish Academy of Sciences, Al. Lotników 32/46, 02-668 Warsaw, Poland \\ ${ }^{4}$ Laboratoire de Physique des Lasers, Atomes et Molécules, UMR 8523 CNRS - Université Lille 1, Bâtiment P5, \\ F-59655 Villeneuve d'Ascq Cedex, France \\ ${ }^{5}$ Laboratoire de Physico-Chimie de l'Atmosphère, EA-4493, Université du Littoral-Côte d'Opale, \\ 59140 Dunkerque, France
}

(Received 16 December 2014; accepted 17 February 2015; published online 13 March 2015)

\begin{abstract}
Polycyclic aromatic hydrocarbons (PAHs) are highly relevant for astrophysics as possible, though controversial, carriers of the unidentified infrared emission bands that are observed in a number of different astronomical objects. In support of radio-astronomical observations, high resolution laboratory spectroscopy has already provided the rotational spectra in the vibrational ground state of several molecules of this type, although the rotational study of their dense infrared (IR) bands has only recently become possible using a limited number of experimental set-ups. To date, all of the rotationally resolved data have concerned unperturbed spectra. We presently report the results of a high resolution study of the three lowest vibrational states of quinoline $\mathrm{C}_{9} \mathrm{H}_{7} \mathrm{~N}$, an $\mathrm{N}$-bearing naphthalene derivative. While the pure rotational ground state spectrum of quinoline is unperturbed, severe complications appear in the spectra of the $v_{45}$ and $v_{44}$ vibrational modes (located at about $168 \mathrm{~cm}^{-1}$ and $178 \mathrm{~cm}^{-1}$, respectively). In order to study these effects in detail, we employed three different and complementary experimental techniques: Fourier-transform microwave spectroscopy, millimeter-wave spectroscopy, and Fourier-transform far-infrared spectroscopy with a synchrotron radiation source. Due to the high density of states in the IR spectra of molecules as large as PAHs, perturbations in the rotational spectra of excited states should be ubiquitous. Our study identifies for the first time this effect and provides some insights into an appropriate treatment of such perturbations. @ 2015 AIP Publishing LLC. [http://dx.doi.org/10.1063/1.4913750]
\end{abstract}

\section{INTRODUCTION}

Polycyclic Aromatic Hydrocarbon (PAH) molecules and their derivatives (cations, dehydrogenated, nitrogen incorporated compounds, ...) are suspected to be present in space and have been proposed as the carriers of the so-called unidentified infrared emission (UIE) bands observed in a large variety of astrophysical objects. ${ }^{1-4}$ However, apart from the detection of benzene by Cernicharo et al.,${ }^{5}$ no unambiguous detection of a single PAH molecule, based on rotationally resolved spectra, has been reported up to date despite several attempts concerning specific species (see, e.g., Refs. 6 and 7). Various alternative suggestions for the UIE carriers have been proposed including relatively large amorphous carbonaceous solids with mixed aromatic/aliphatic structures. ${ }^{8}$ Nevertheless, it is likely that, as products of combustion, PAHs and their derivatives exist in space and recent empirical calculations suggest that the rotational spectrum of slightly asymmetric planar PAHs would be suitable for radio astronomical searches. ${ }^{9}$ In this context, it is obvious that accurate laboratory studies of

\footnotetext{
a) Present address: Harvard - Smithsonian Center for Astrophysics, 60 Garden Street, MS-50, Cambridge, Massachusetts 02138, USA.
}

rotationally resolved spectra are mandatory in order to support astronomical observations. The increased detector sensitivity, spectral resolution, and spectral coverage provided by recently developed powerful observational platforms such as ALMA (Atacama Large Millimeter Array) enable the detection of new molecules in their ground state (GS) and, in the case of relatively abundant species, within some of their vibrational excited states (ES). It is therefore relevant for astrophysics to provide both GS and ES pure rotational frequencies for polar PAH species. To date, laboratory measurements have mostly been limited to the GS pure rotational spectra of PAHs and their nitrogen containing heterocycles (defined as PANHs) obtained from high resolution microwave techniques. ${ }^{10-15}$ Very recently, new infrared (IR) techniques have enabled studies of the rotational structures of vibrationally excited states of a few PAH molecules and other relatively large Cbearing molecules. ${ }^{16-19}$

The GS of most planar PAHs should not be affected by any ro-vibrational interaction since the lowest vibrations are out-of-plane deformations which lie several $\mathrm{THz}$ above the GS (see, e.g., Malloci et al. ${ }^{20}$ for a spectroscopic database of PAHs). Due to the presence of these low-lying vibrational modes, the density of states is rapidly increasing (see, 
e.g., Basire $e t$ al. ${ }^{21}$ for calculations of the density of states in large molecular systems) and should in contrast lead to numerous perturbations in the ES rotational structures. To date, very few spectroscopic studies of large molecules deal with rotationally perturbed spectra; and even in the case of monocyclic species, perturbations of the ES have been ignored in the rotational analysis (see, e.g., Esselman et al. ${ }^{22}$ ). The purpose of the present study is thus to provide a thorough understanding of the rotational structure of the two lowest vibrational modes of quinoline $\mathrm{C}_{9} \mathrm{H}_{7} \mathrm{~N}\left(v_{45}\right.$ and $\left.v_{44}\right)$, a simple two ring PANH molecule, using complementary high resolution experimental techniques. Quinoline is a prolate asymmetric top with a relatively large permanent dipole moment $\left(\mu_{\mathrm{a}}\right.$ $=0.14 \mathrm{D}$ and $\mu_{\mathrm{b}}=2.01 \mathrm{D}$, from density functional theory (DFT) calculations) for which GS pure rotational transitions (b-type) were recorded and analyzed by Kisiel et al., ${ }^{11}$ where it was also noted that rotational transitions in lowest vibrational states were heavily perturbed. The vibrational IR spectrum has been analyzed in several papers (see, e.g., Wait and McNerney ${ }^{23}$ ), and has recently been extended to the far-infrared (far-IR) ${ }^{24}$ In this last paper, Martin-Drumel et al. assigned the lowest vibrational mode, $v_{45}$, to the unresolved $c$-type band centered at about $168 \mathrm{~cm}^{-1}$ (the calculated intensity of the $v_{45}$-GS band is $4.6 \mathrm{~km} \mathrm{~mol}^{-1}$ ) while the much weaker $v_{44}$-GS remained unidentified (calculated intensity of $0.2 \mathrm{~km} \mathrm{~mol}^{-1}$ ).

The present paper reports the results of a comprehensive investigation of the rotational structure of the three lowest vibrational states of quinoline (GS, $v_{45}$ and $v_{44}$ ) using three complementary experimental techniques. The spectral analysis enabled the identification of considerable perturbations between the two excited states requiring a specific treatment in the fit of the effective Hamiltonian parameters. The laboratory techniques used to record the different spectra and the details of the anharmonic DFT calculations performed to facilitate the preliminary analysis are described in Sec. II; Sec. III details the analysis procedure; and finally, Sec. IV contains (i) the results of the fit using a dedicated Hamiltonian to treat the interacting states and (ii) the discussion of the possible generalization of such spectral complications to other PAH molecules.

\section{LABORATORY TECHNIQUES}

We made use of three complementary techniques to obtain a thorough spectroscopic coverage of the rotational structures for the three lowest vibrational states of quinoline (GS, $v_{45}$ and $\left.v_{44}\right)$.

\section{A. Synchrotron-based Fourier-transform far-IR spectroscopy}

A synchrotron-based Fourier transform (FT) spectrum was recorded on the AILES beamline of the SOLEIL synchrotron in the far-IR spectral region by using a roomtemperature long pathlength absorption cell connected to a high resolution IFS125 Bruker interferometer. The operating mode was described in detail in previous articles (see, e.g., Ref. 25). The absorption pathlength was set to about $150 \mathrm{~m}$, a liquid He cooled Si-bolometer detector and a $6 \mu \mathrm{m}$ thick mylar beamsplitter were used. The synchrotron radiation extracted by the AILES beamline was used as the far-IR continuum source (details of the performances of the source for the far-IR spectroscopy are given in Ref. 26). Quinoline at a pressure of about $1.5 \mathrm{~Pa}$ (purchased from Sigma-Aldrich and used without further purification) was introduced into the cell. The spectrum was recorded at a $0.001 \mathrm{~cm}^{-1}$ resolution over the $50-300 \mathrm{~cm}^{-1}$ region (limited by the optical filter of the bolometer). We coadded 400 interferograms to optimize the signal-to-noise ratio (SNR). Far-IR water absorption lines allowed accurate frequency calibration of the spectrum. ${ }^{27}$ In these conditions, we observe the $v_{45}$-GS $c$-type band of quinoline centered at about $168 \mathrm{~cm}^{-1}$. The band is composed of an intense and unresolved ${ }^{Q} Q$-branch and partially rotationally resolved ${ }^{P} P$-and ${ }^{R} R$-branches. A closer look at the $R$-branch region while degrading the resolution to $0.01 \mathrm{~cm}^{-1}$ reveals the very weak $v_{44}$-GS $Q$-branch (see Figure 1 ). The detection of this $Q$-branch allows a rough estimate of the $v_{45}-v_{44}$ energy difference (of about $9.4 \mathrm{~cm}^{-1}$ ), this value being the key parameter to begin the perturbation analysis of the $v_{45}$ and $v_{44}$ rotational structures. As a consequence of the large spectral congestion and relatively low SNR (about 10 for the most intense lines), the frequency measurement accuracy of the FT-FIR lines is estimated to be $0.0002 \mathrm{~cm}^{-1}$ (about $6 \mathrm{MHz}$ ).

\section{B. Millimeter wave spectroscopy}

The most extensive segment of the pure rotational spectrum of quinoline was recorded in the frequency range 140-220 GHz at the LPCA laboratory of Dunkerque University by using a sub-millimeter (sub-mm) spectrometer described in detail in Ref. 28. Briefly, a post-amplified synthesizer (7.7-12.2 $\mathrm{GHz}$ ) drives a commercial frequency multiplier source from Virginia Diodes, Inc., to cover the frequency range between 50 and $930 \mathrm{GHz}$. Two off-axis paraboloid mirrors enable first to collimate the emitted polarized radiation into the single path absorption cell and then to focus the output beam onto the detector, an InSb liquid He-cooled bolometer from QMC Instruments. A $1.25 \mathrm{~m}$ long stainless-steel absorption-cell equipped with Teflon windows was used in this experiment.

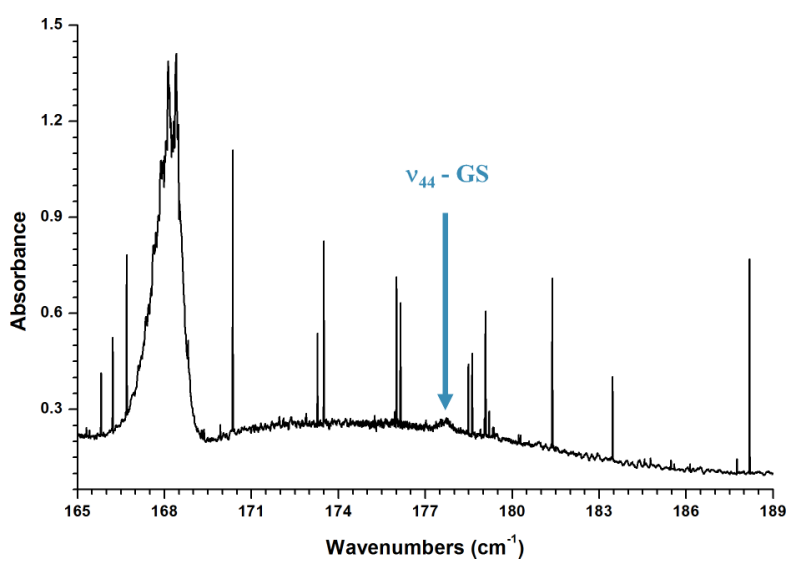

FIG. 1. Detection of the weakly IR active $v_{44}$-GS band in the $R$-branch region of the $v_{45}$-GS band. The spectrum was obtained by degrading the spectral resolution to $0.01 \mathrm{~cm}^{-1}$. At this resolution, water lines appear as sharp features superimposed on the continuum of absorption. 
A constant flow, ensured by a turbomolecular pump, of $1 \mathrm{~Pa}$ of quinoline was used to record the spectrum. The spectrum was recorded using a $25 \mathrm{kHz}$ frequency modulation, a $20 \mathrm{~ms}$ time constant, $30 \mathrm{kHz}$ steps, and a second harmonic lockin detection. In these conditions, we observe $b$-type pure rotational lines within the GS and ES (in particular $v_{45}$ and $v_{44}$ ) with a frequency accuracy estimated to $30 \mathrm{kHz}$.

As the analysis progressed, the spectral coverage has been extended to several specific regions predicted to contain highly perturbed lines. A $222-238 \mathrm{GHz}$ segment and multiple smaller windows at frequencies ranging from 282 to $298 \mathrm{GHz}$ were thus recorded by using a backward-wave oscillator based millimeter-wave (mm-wave) spectrometer in Warsaw, ${ }^{29,30}$ providing an estimated frequency accuracy of $30 \mathrm{kHz}$.

\section{Fourier-transform microwave spectroscopy}

The jet-cooled spectrum of quinoline was recorded in the frequency range $4-20 \mathrm{GHz}$ at the PhLAM laboratory (in Lille) using a supersonic expansion Fourier-transform microwave (FTMW) spectrometer (a detailed description of the apparatus can be found in Refs. 31 and 32). Quinoline vapor was mixed with the carrier gas and expanded through a heated pulsed nozzle ${ }^{33}$ operating at a backing pressure of $10^{5} \mathrm{~Pa}$ of carrier gas, a temperature of $413 \mathrm{~K}$, and a repetition rate of $1.5 \mathrm{~Hz}$. The best SNR was obtained by using $\mathrm{Ne}$ as buffer gas in preference to the other commonly used buffer gases $\left(\mathrm{He}, \mathrm{Ar}\right.$ and $\mathrm{N}_{2}$ ). In such conditions, the supersonic expansion ensures a rather efficient rotational cooling together with a less efficient vibrational cooling, allowing the lowlying vibrational states to be sufficiently populated. This, combined with intense signals (the projection of the permanent electric dipole moment of quinoline along the $b$ inertial axis is estimated to about 2 Debye) and the high sensitivity of the spectrometer, enables the observation of transitions within these states, with intensities about a thousand times weaker than the corresponding GS transitions. Signals were accumulated 500 to 1000 times to obtain a relatively high SNR. In complement to the GS $b$-type transitions reported by Kisiel et al., ${ }^{11}$ the small contribution of the permanent dipole moment along the $a$ inertial axis (about $0.2 \mathrm{D}$ ) permits the observation of some GS $a$-type transitions. Each resonance frequency was determined as the average frequency of the two Doppler components (due to the coaxial arrangement of the jet and the Fabry-Perot cavity) and the uncertainty of the measurements is estimated to be $4 \mathrm{kHz}$.

Complementary room-temperature measurements were also performed with a 8-18 GHz waveguide FTMW spectrometer developed in the Kiel laboratory ${ }^{34,35}$ and currently located in Warsaw allowing estimated uncertainty of $10 \mathrm{kHz}$. Such measurements complete the coverage of quantum number values in the bridging region between those accessible in the supersonic expansion FTMW and in the room-temperature mw-wave spectra.

\section{Computational details}

Calculations were conducted using the Gaussian 09 software package ${ }^{36}$ on the SOLEIL cluster. The theoretical approach (at the B97-1/cc-pVTZ//ANO-DZP level of computation) has already been described elsewhere and resulted in accurate predictions of the spectroscopic constants for naphthalene $\mathrm{e}^{19}$ and 18 others PAHs and PANHs. ${ }^{32,37}$ The rotational analysis of the two ES of quinoline presented hereafter is another validation of this approach.

\section{SPECTRAL ANALYSIS}

The analysis of the relatively complex rotational structure of the $v_{45}$ and $v_{44}$ ES was performed in a multi-step procedure. At every step of the process, we made use of the Loomis-Wood assignment technique available in the $\mathrm{LWWa}^{38}$ and AABS packages ${ }^{39,40}$ for line by line assignment. The SPFIT/SPCAT program suite of Pickett ${ }^{41}$ was used to construct the Hamiltonian, fit the molecular parameters, and calculate the energy levels.

In a first step, by making use of the highly reliable DFT ES rotational constants (see Ref. 37 for a detailed comparison of experimental vs. DFT rotational constants for PAHs species in their GS and ES), we assigned the $v_{45}$-GS ro-vibrational band obtained using the FT-FIR set-up. This unambiguous analysis, based on accurate GS combination differences calculated from the rotational constants of Ref. 11, provided experimental values of the $v_{45}$ energy, $A^{\prime}, B^{\prime}, C^{\prime}$ rotational constants and quartic centrifugal distortion parameters (denoted hereafter cd-terms) at the IR accuracy. At this stage, no perturbation of the rotational pattern in the $c$-type transitions was detected in the FT-FIR spectrum. As for previous studies of comparable species, the analysis was limited to ${ }^{P} P$ and ${ }^{R} R$ branches of the $v_{45}$-GS band. Figure 2 shows portions of the $v_{45}$-GS experimental spectrum in the $P_{-}, Q-$, and $R$-regions with corresponding simulated spectra obtained from the final set of parameters (see later in the text) using the PGOPHER program. ${ }^{42}$ The top trace shows the $Q$-branch region of the $v_{45}$ GS band. This unresolved feature is composed of $Q$-branches of the fundamental band and numerous hot bands involving several quanta of the low-lying vibrational states of quinoline. The middle and lower traces show the rotationally resolved lines in the $P$ - and $R$-branches, respectively. Due to spectral congestion and the presence of numerous hot band lines, the comparison between experimental and calculated spectra is not straightforward. Only the most intense transitions in the simulation are clearly visible on the experimental traces. As detailed further in the text, 3578 FT-FIR transitions were included in the global dataset and fitted to the experimental accuracy $\left(0.0002 \mathrm{~cm}^{-1}\right)$.

In a second step, we analyzed the pure rotational $b$-type mm-wave transitions within $v_{45}$ and $v_{44}$. The assignment of the $v_{45}$ pure rotation spectrum was based first on the approximate set of constants obtained from the FT-FIR spectrum. Then, series of lines displaying similar relative intensities were assigned to the pure rotation spectrum of $v_{44}$ with the support of the calculations. The perturbation patterns were accounted for in a step by step process. Figure 3 illustrates a typical repetitive feature in the rotational spectrum of quinoline, which is a type-II band characteristic of high- $J$ spectra of near-planar molecules ${ }^{43,44}$ and consisting of transitions with decreasing values of $J$ and increasing values of $K_{a}$ in the 

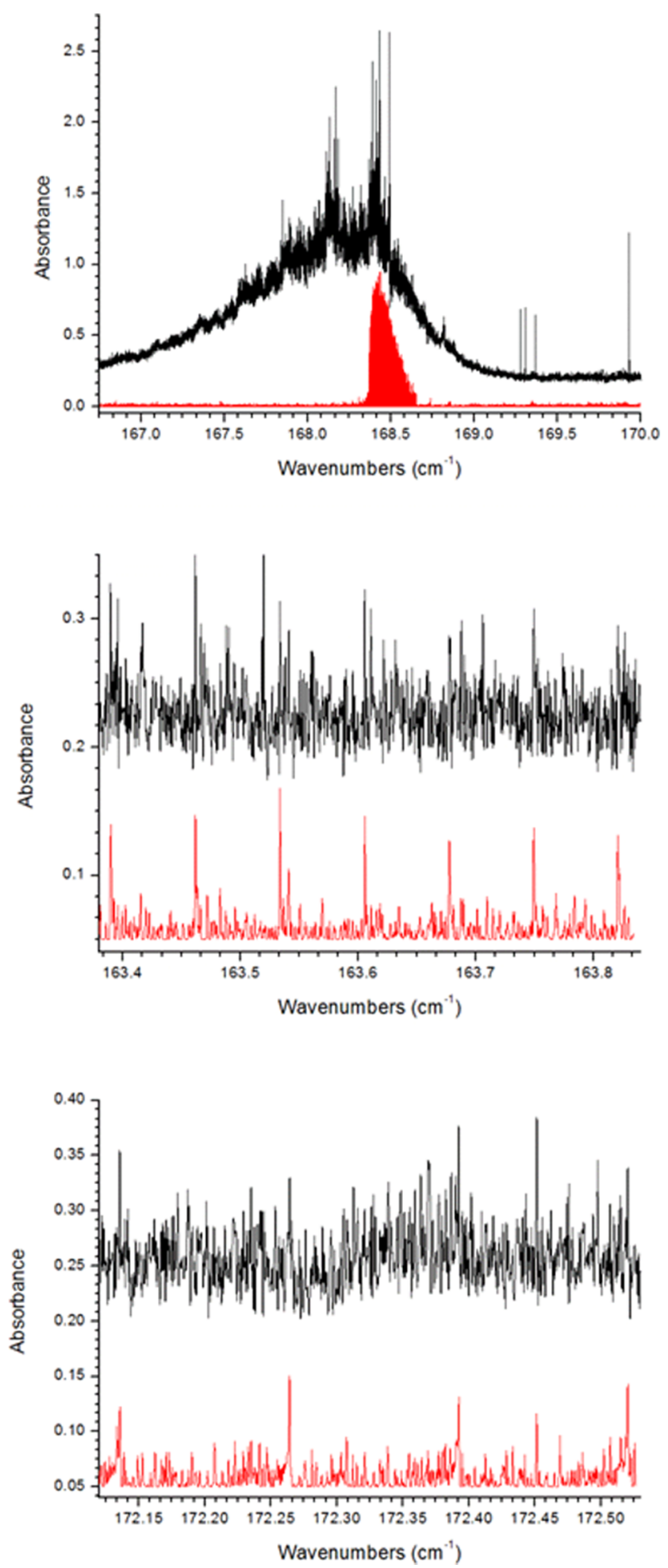

FIG. 2. Zoom into the $Q$-, $P$-, and $R$-regions of the $v_{45}$-GS band, from top to bottom. Black upper traces correspond to the FT-FIR experimental spectrum and red lower traces are the simulated spectra obtained from the final set of fitted parameters. The intensity scale corresponds to absorbance in the experimental spectrum.

direction away from the bandhead. Some smoothing and filtering of the experimental trace was performed using the dedicated programs available on the PROSPE website. ${ }^{45,46}$ The simulation was made using predictions from the SPCAT program based on the final set of parameters and contains the $b$-type rotational lines calculated for the GS, $v_{45}$, and $v_{44}$.

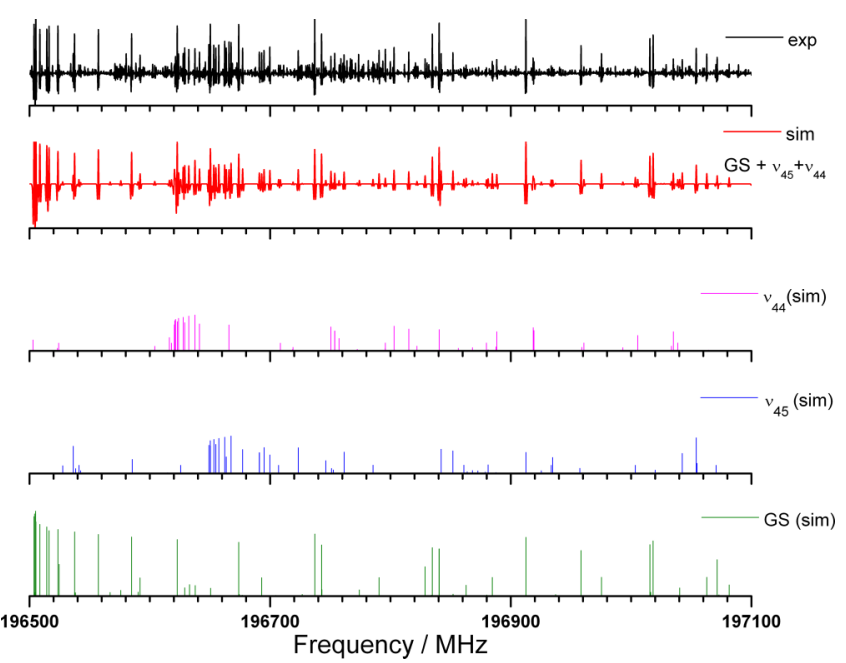

FIG. 3. Relatively narrow frequency range $(600 \mathrm{MHz})$ of the pure rotational spectrum of quinoline around $197 \mathrm{GHz}$. The experimental spectrum is compared to a simulation accounting for the contribution of pure rotational transitions within the GS, $v_{45}$, and $v_{44}$ calculated using the final set of fitted parameters.

The agreement between the observed and the simulated spectra is quite good, both in frequency and in relative intensity. Numerous lines present in the experimental trace are still not accounted for in the simulation: these lines correspond to pure rotational transitions within higher vibrational ES, for example, within the two fundamental modes $v_{31}\left(A^{\prime}\right.$ symmetry and energy of about $375 \mathrm{~cm}^{-1}$, Ref. 24) and $v_{43}\left(A^{\prime \prime}\right.$ symmetry and energy of about $389 \mathrm{~cm}^{-1}$, Ref. 24), as well as within $2 v_{45}, 2 v_{44}$, and $v_{45}+v_{44}$. The rotational analysis of transitions involving this stack of states is beyond the scope of the present study; however, we noticed that the rotational patterns of these states seem highly perturbed and reflect the increasing complexity of such studies when proceeding to higher ES of relatively large molecules.

Many considerable perturbations in the pure rotational mm-wave spectra of $v_{45}$ and $v_{44}$ were identified and were reasonably quickly found to be characterized by the difference $\Delta K_{a}=2$ between perturbing pairs of rotational sublevels in the two vibrational states. Description of such perturbations with a suitable Hamiltonian (see further below) is in the form of closed matrices for a given value of $J$. Perturbation contributions in such matrices arise from off-diagonal terms and, when the matrices are diagonalized to determine the eigenvalues, the perturbation effects contribute in equal magnitude, but in opposite sign increments to the eigenvalues. This has the consequence that plots of transition sequences for specific values of $K_{a}$ made as a function of $J$ should display mirror image character for the correctly identified perturbation partners. Figure 4 illustrates collected examples of such perturbation plots for the lowest values of $K_{a}$. The scaled amplitude of the most pronounced interaction in this plot (between $K_{a}{ }^{\prime \prime}=13$ of $v_{45}$ and $K_{a}{ }^{\prime \prime}=11$ of $v_{44}$ ) is $18 \mathrm{MHz}$ at $J^{\prime \prime}+1=97$, corresponding to a considerable perturbation shift of $1746 \mathrm{MHz}$. Plots of this type can be readily constructed with Loomis-Wood techniques since they only require confident assignment of transition sequences, although the apparent zig-zag behaviour eventually needs to be 


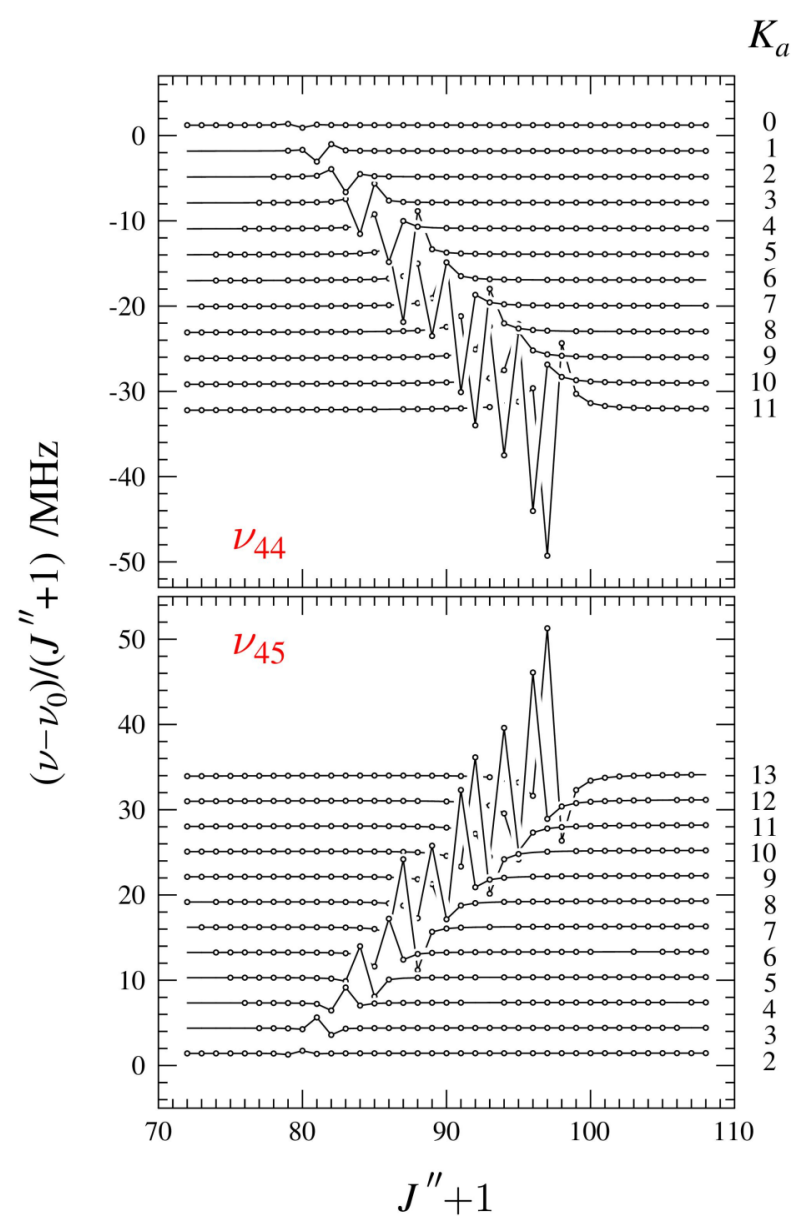

FIG. 4. Illustration of the mirror image dependence of the $\Delta K_{a}=2$ perturbations observed between the pure rotation transitions in $v_{45}$ and $v_{44}$. The plots are of simple scaled frequency difference between the frequency of a given excited state transition and its counterpart in the unperturbed GS. Continuous lines are from the final fit, while the markers identify experimental values and there are no discernable differences between them.

confirmed by a suitable fit. This type of interaction is observed for all $K_{a}{ }^{\prime \prime}$ values and its amplitude exceeds several $\mathrm{GHz}$ in cases involving high $K_{a}{ }^{\prime \prime}$ values.

An additional powerful tool for graphic visualization of the perturbations expected over the whole range of quantum numbers accessible to experiment is to plot the quantity
(1- $\left.P_{\text {mix }}\right)$, where $P_{\text {mix }}$ is the mixing coefficient for a given ro-vibrational level. ${ }^{47,48}$ The mixing coefficient $P_{\text {mix }}$ reflects the eigenvalue purity and is available in the output from the SPCAT program (in the energy file). The quantity $\left(1-P_{\text {mix }}\right)$ will therefore describe the amplitude of mixing between the rotational levels belonging to different vibrational states. Figure 5 presents such a plot for the $v_{45}$ and $v_{44}$ energy levels. The red circles denote the rotational levels with the largest mixing magnitudes.

Since the perturbation takes place between rotational sublevels for the two vibrational states characterized by different values of $K_{a}$ but the same value of $J$, we can easily identify in both parts of Figure 5 similarly shaped regions of perturbation, in the form of a two-pronged pattern, identified with (a). The higher- $J$ prong results from the $\Delta K_{a}=2$ interaction observed extensively for all $K_{a}$ values in the limit of the continuous mm-wave coverage up to $238 \mathrm{GHz}$ (already partly shown on Fig. 4). The lower- $J$ more vertical prong corresponds to $\Delta K_{a}=2$ perturbations for higher values of $K_{a}$ (starting from $K_{a}{ }^{\prime \prime}=36$ for $v_{45}$ and $K_{a}{ }^{\prime \prime}=34$ for $v_{44}$ ), which were probed by the less extensive measurements at 282-298 GHz. One can also discern several other perturbation regions, which only appear for one of the vibrational states and do not have a counterpart in the other. The most prominent examples are marked in Figure 5 by (b) and arise from neardegeneracy of rotational states with the same value of $K_{c}$. This degeneracy comes in at high $J$ and takes place within a given submatrix of the Hamiltonian as factorized by SPCAT, so that such levels are often identified by SPCAT as mixed. Such $\left(1-P_{\text {mix }}\right)$ plots enable targeting of specific transitions in the experimental spectrum that have particular sensitivity to the perturbations and thus allow improved determination of the coupling parameters.

As a final step in the rotational analysis of the $v_{45}$ and $v_{44}$ ES, we recorded pure rotational (b-type) transitions within both ES by using a supersonic expansion FTMW apparatus, and a room-temperature waveguide FTMW. These measurements resulted in very accurate $(4 \mathrm{kHz}$ and $10 \mathrm{kHz}$, respectively) hyperfine-resolved frequencies for the lowest $J^{\prime \prime}$ and $K_{a}{ }^{\prime \prime}$ transitions. The measured lines were introduced into the fit and provided a very reliable final set of constants for both ES.

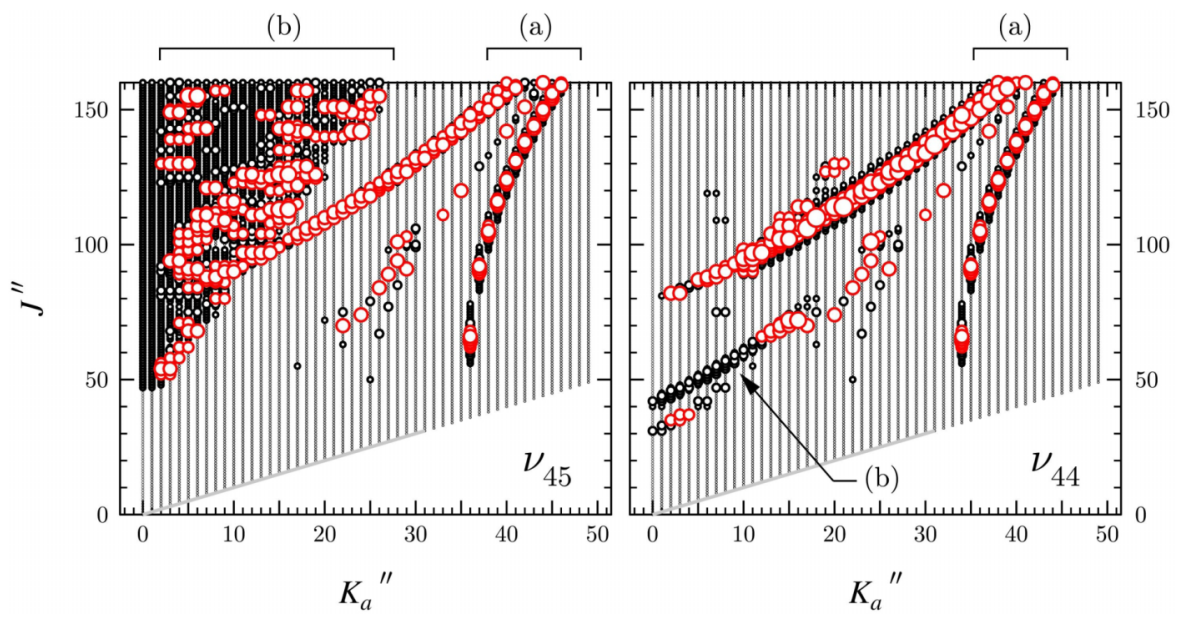

FIG. 5. The plot of values of $\left(1-P_{\text {mix }}\right)$ for the rotational energy levels of $v_{45}$ (left) and $v_{44}$ (right) vibrational states. The circle diameter indicates the magnitude of $\left(1-P_{\text {mix }}\right)$. The red circles show the largest mixing values, which allow easy identification of quantum numbers of the many possible level crossing perturbations. 

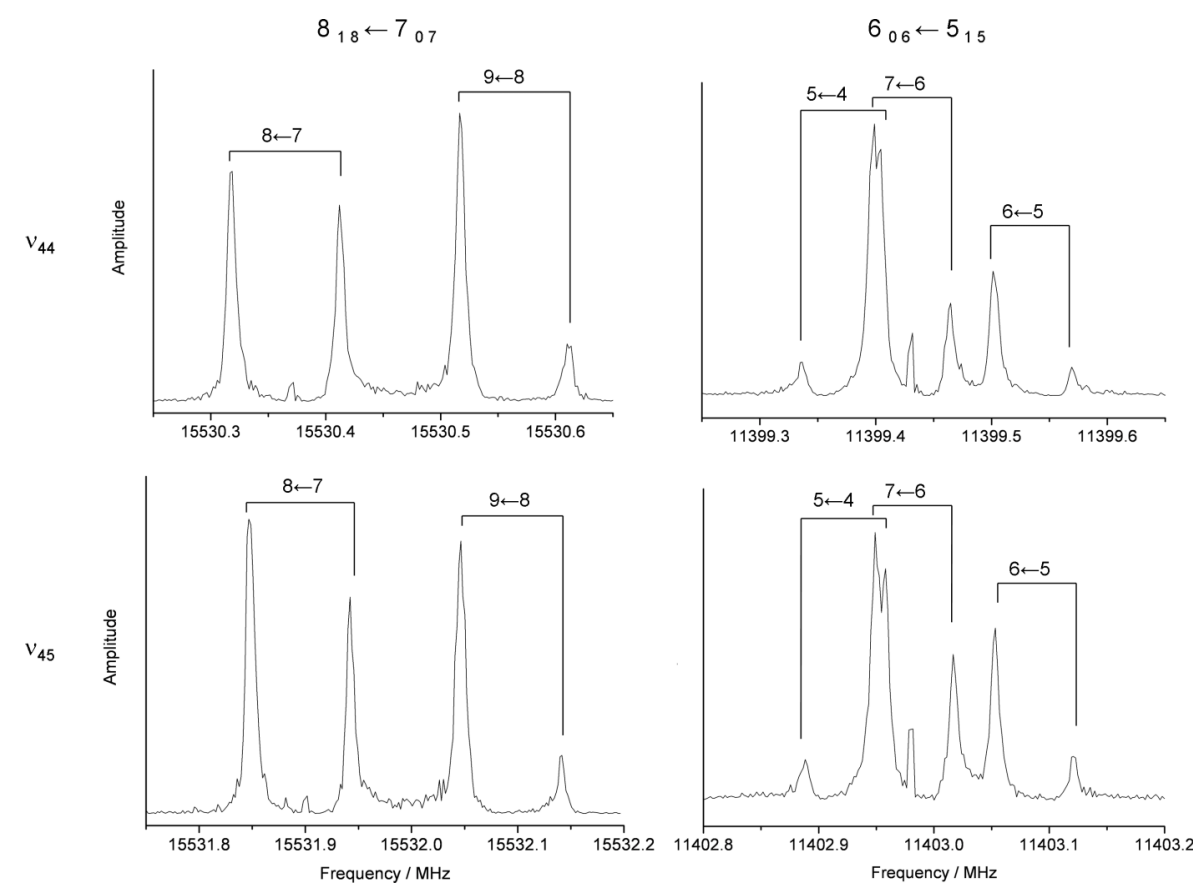

FIG. 6. Examples of pure rotational lines (in the form of Doppler doublets) for the two excited states of quinoline recorded by supersonic expansion FTMW spectroscopy. The lines exhibit a hyperfine structure due to the nitrogen nuclear quadrupole coupling. Assignments are in the format $J_{K a^{\prime} K c^{\prime}}^{\prime}$ $\leftarrow J_{K a^{\prime \prime} K c^{\prime \prime}}^{\prime \prime}$ and $F^{\prime} \leftarrow F^{\prime \prime}$.
Figure 6 shows lines involving $K_{a}{ }^{\prime \prime}=0$ and 1 recorded in a jet expansion. The hyperfine structure in both ES due to the ${ }^{14} \mathrm{~N}$ nuclear quadrupole hyperfine coupling is clearly resolved. Although a dependence of the quadrupole coupling on the vibrational excitation has been demonstrated (see, e.g., Bechtel et $a .^{49}$ ), no significant changes in the hyperfine parameters were observed in the present case of quinoline as for methyl halides ${ }^{50}$ and some diatomic molecules. ${ }^{51}$ Since among the about ten thousand lines in the dataset, only the several tens of FTMW lines exhibit resolved hyperfine structure, two different methods have been used to include these lines in the fit. For the hyperfine-free method (see Fits I and III below), hyperfine-free central frequencies for the experimental FTMW lines were obtained by using the set of parameters derived from the best fit of only the FTMW lines of a given state (GS, $v_{44}$, or $v_{45}$ individually) and then setting the hyperfine constants to zero. This method aimed at simplifying the fit by removing the hyperfine parameters which are only sensitive to the several tens of FTMW lines. For the hyperfine method (see Fits II and IV below), all hyperfine components of the FTMW lines were used as measured and the relevant hyperfine parameters fitted. In this case, the Hamiltonian is closer to the physical properties of the system. The results obtained are discussed in Sec. IV.

\section{DISCUSSION}

Both $v_{45}$ and $v_{44}$ states are of $A^{\prime \prime}$ symmetry (out-ofplane modes) and can thus interact through $c$-type Coriolis interaction and Fermi resonance. Therefore, we used the following model to fit the experimental transitions involving the GS (isolated state) and both $v_{45}$ and $v_{44}$.

The pure rotational part of the Hamiltonian is the Watson's $A$-reduced effective asymmetric rotor Hamiltonian in the $I^{r}$ representation (Ref. 52) as described by the following equation:

$$
\begin{aligned}
H_{\mathrm{rot}}^{v}= & {\left[A-\frac{1}{2}(B+C)\right] P_{z}^{2}+\frac{1}{2}(B+C) P^{2} } \\
& +\frac{1}{2}(B-C)\left(P_{x}^{2}-P_{y}^{2}\right)-\Delta_{J} P^{4} \\
& -\Delta_{J K} P^{2} P_{z}^{2}-\Delta_{K} P_{z}^{4}-\delta_{K}\left[P_{z}^{2}, P_{x}^{2}-P_{y}^{2}\right]_{+} \\
& -2 \delta_{J} P^{2}\left(P_{x}^{2}-P_{y}^{2}\right)+\cdots .
\end{aligned}
$$

As found below, the rotational part of the Hamiltonian can be limited to the rotational constants $A, B$, and $C$, and the quartic cd-terms.

In order to account for the Coriolis and Fermi interactions observed in the $v_{45} / v_{44}$ dyad, we used the following $2 \times 2$ block Hamiltonian:

$$
\left(\begin{array}{cc}
H_{\mathrm{rot}}^{45} & H_{\mathrm{cor}}^{(45,44)}+H_{F}^{(45,44)} \\
H_{\mathrm{cor}}^{(45,44)}+H_{F}^{(45,44)} & H_{\mathrm{rot}}^{44}+\Delta E\left(v_{44}-v_{45}\right)
\end{array}\right) .
$$

The diagonal terms of this Hamiltonian consist of (i) the pure rotational Hamiltonian as already defined above and (ii) the energy difference between $v_{44}$ and $v_{45}$. The off-diagonal elements are the sum of the Coriolis and Fermi terms. In the present case, the leading terms for the Coriolis interaction result from the $H_{21}$ and $H_{22}$ terms of the vibration-rotation Hamiltonian as described in Ref. 53. The Coriolis terms are expressed as

$$
\begin{aligned}
H_{\mathrm{cor}}^{(45,44)}= & i\left(G_{c}+G_{c}^{J} P^{2}+G_{c}^{K} P_{z}^{2}+\ldots\right) P_{y} \\
& +\left(F_{a b}+\cdots\right)\left(P_{z} P_{x}+P_{x} P_{z}\right) .
\end{aligned}
$$

The second type of off-diagonal elements arises from Fermi resonance and are expressed as follows:

$$
\begin{aligned}
H_{F}^{(45,44)}= & W_{F}+W_{F}^{J} P^{2}+W_{F}^{K} P_{z}^{2}+\cdots \\
& +\left[\left(W_{ \pm}+W_{ \pm}^{J} P^{2}+W_{ \pm}^{K} P_{Z}^{2}+\cdots\right), P_{x y}^{2}\right]_{+}+\cdots,
\end{aligned}
$$

where $P_{x y}^{2}=P_{x}^{2}-P_{y}^{2}$ and $[A, B]_{+}=A B+B A$. 
TABLE I. Summary of the experimental dataset obtained from the different experiments and details of the fit. The RMS values are similar for the four fits realized in the present study; they are expressed in $\mathrm{MHz}$ and $\mathrm{cm}^{-1}$ for the microwave and IR lines, respectively.

\begin{tabular}{lccccc}
\hline \hline & $\begin{array}{c}\text { Lines } \\
\text { fitted }\end{array}$ & RMS & $\begin{array}{c}\text { RMS } \\
\text { error }\end{array}$ & $\begin{array}{c}J^{\prime \prime} \\
\text { range }\end{array}$ & $\begin{array}{c}K_{a}{ }^{\prime \prime} \\
\text { range }\end{array}$ \\
\hline $\mathrm{GS}$ & 258 & $0.028 \mathrm{MHz}$ & 0.95 & $1-143$ & $0-58$ \\
$v_{45}$ & 3012 & $0.048 \mathrm{MHz}$ & 0.81 & $2-148$ & $0-58$ \\
$v_{44}$ & 2999 & $0.048 \mathrm{MHz}$ & 0.81 & $1-130$ & $0-58$ \\
$v_{45}-\mathrm{GS}$ & 3578 & $0.0002 \mathrm{~cm}^{-1}$ & 1.02 & $9-114$ & $9-60$ \\
\hline \hline
\end{tabular}

The results of the fit for the four different data subsets, pure rotational transitions for each vibrational state, and vibration-rotation transitions are listed in Table I. These results are obtained from a simultaneous fit of all the experimental lines weighted according to their experimental accuracy. In total, 9847 lines have been included in the fit involving $J^{\prime \prime}$ and $K_{a}{ }^{\prime \prime}$ values up to 148 and 60, respectively. For each subset of data, the RMS values are in good agreement with the experimental accuracy as reflected by the RMS error of close to unity.

\section{A. General description of the fitted parameters}

The present dataset allowed determination of 33 GS and ES vibration-rotation parameters as well as 3 hyperfine parameters when the hyperfine structure is accounted for (hyperfine constants for GS and ES assumed to be equal). Apart from the hyperfine-free and hyperfine-inclusive methods introduced above, we also have used two different fitting approaches concerning the treatment of rotational constants, leading to four different fits, all in Watson's $A$ reduction. In the first approach (Fit I and Fit II), a straightforward fit of the rotational constants was performed (Fit I: hyperfine-free/Fit II: hyperfine-inclusive), while in the second approach linear combinations of the rotational constants (denoted hereafter $A B C$ ) was performed (Fit III: hyperfine-free/Fit IV: hyperfineinclusive). Determination of the sum of the rotational constants for the ES turned out to be very precise, while that of their difference is much poorer and is the reason for the degradation in the uncertainties estimated for all rotational constants in Fits I and II. It is noteworthy that there is not much difference between the fits obtained with and without inclusion of the resolved hyperfine structure (comparisons Fit I/Fit II, and Fit III/Fit IV). For all fits, the GS parameter values and their accuracies are very similar to the values obtained from Kisiel et al. ${ }^{11}$ (who limited the study to the GS pure rotational $b$-type transitions). Most of the differences between the fits concern uncertainties in fitted parameters resulting from unitary intercorrelations between specific ES parameters as discussed further in this section. Since these molecules are relatively rigid rotors, the quartic cd-terms remain small and the fits did not require inclusion of any of the higher order cdterms, as also found in all previous studies of the ES rotational structure of PAHs (see Ref. 37 for a review concerning the rotationally resolved dataset of PAHs and derivatives). The parameters obtained with fits II and IV are given in Table II and in the supplementary material. ${ }^{56}$ Among these fits, we prefer Fit IV which uses linear combination of $A B C$ and also successfully accounts for the hyperfine structure in GS and both ES.

Inclusion of both Coriolis and Fermi terms was mandatory in order to account for the perturbations observed in many rotational sublevels. We fitted the leading $G_{c}$ term in the Coriolis coupling series as well as the higher order terms $G_{c}^{J}$ and $G_{c}^{K}$. The higher order Coriolis parameters in the $F_{a b}$ series were not needed in the fit. As for the Fermi terms, $W_{F}$ and its $J$ and $K$ dependence were fitted. In similarity to the work of Krasnicki et al. ${ }^{54}$ it was possible to complete the model with the higher order $W_{ \pm}^{K}$ parameter of the $W_{ \pm}$ sequence, which allowed the highly accurate, low quantum number value FTMW lines to be encompassed by the fit.

\section{B. Intercorrelations}

As in many similar cases, near unitary intercorrelations exist between the fitted vibrational energies and the leading Fermi term. However, the effective energy difference between the interacting states is actually determined very precisely. This has been discussed in detail in Ref. 55 where actual uncertainties were estimated using error propagation inclusive of the off-diagonal terms in the variance-covariance matrix connecting the Fermi and the vibrational terms. Effective vibrational energies (obtained by diagonalization of the $2 \times 2$ matrix of Eq. (5) in Ref. 55) are listed in Table III. The results of the direct fit of the three highly correlated parameters are given in the first three rows of this table, while the results of reduction to the effective parameters are in the last four rows. In addition, the observation in the mm-wave spectrum of some interstate transitions resulting from perturbation induced state mixing significantly improved the accuracy of the $v_{45^{-}}$ $v_{44}$ energy difference $\left(\Delta E_{(45-44)}\right)$ and lowered correlations between the perturbative terms and the vibrational energies. This final $\Delta E_{(45-44)}$ value of $9.393282(4) \mathrm{cm}^{-1}$ is actually quite close to the estimate obtained from the detection of the very weak Q branch of the $v_{44}-$ GS band in the FT spectrum.

One should note that the ES values of the $\Delta_{K}$ cd-term constants straddle the GS value. Various attempts to combat this effect within the performed fits have been unsuccessful and we conclude that this is due to factors outside the model. One possibility would be that the energy levels of the studied states reaching more and more into the dense structure of states above them, which is a $K_{a}$ dependent effect that can be reflected by effective contributions to constants such as $\Delta_{K}$.

\section{Comparison with anharmonic DFT calculations}

The results of the fit are compared to anharmonic DFT calculations in Table IV. As already described in Ref. 37, the calculated rotational constants for the GS and ES are in good agreement with the experimental results. In the GS, the experimental and the calculated quartic cd-terms and hyperfine structure parameters are also in very good agreement (differences lower than 3\% for the cd-terms), which provides another validation of the calculated quinoline force field. 
TABLE II. List of the parameters (in MHz) obtained from the global fit of all experimental values. Values of the parameters kept fixed in the fit are indicated in square brackets.

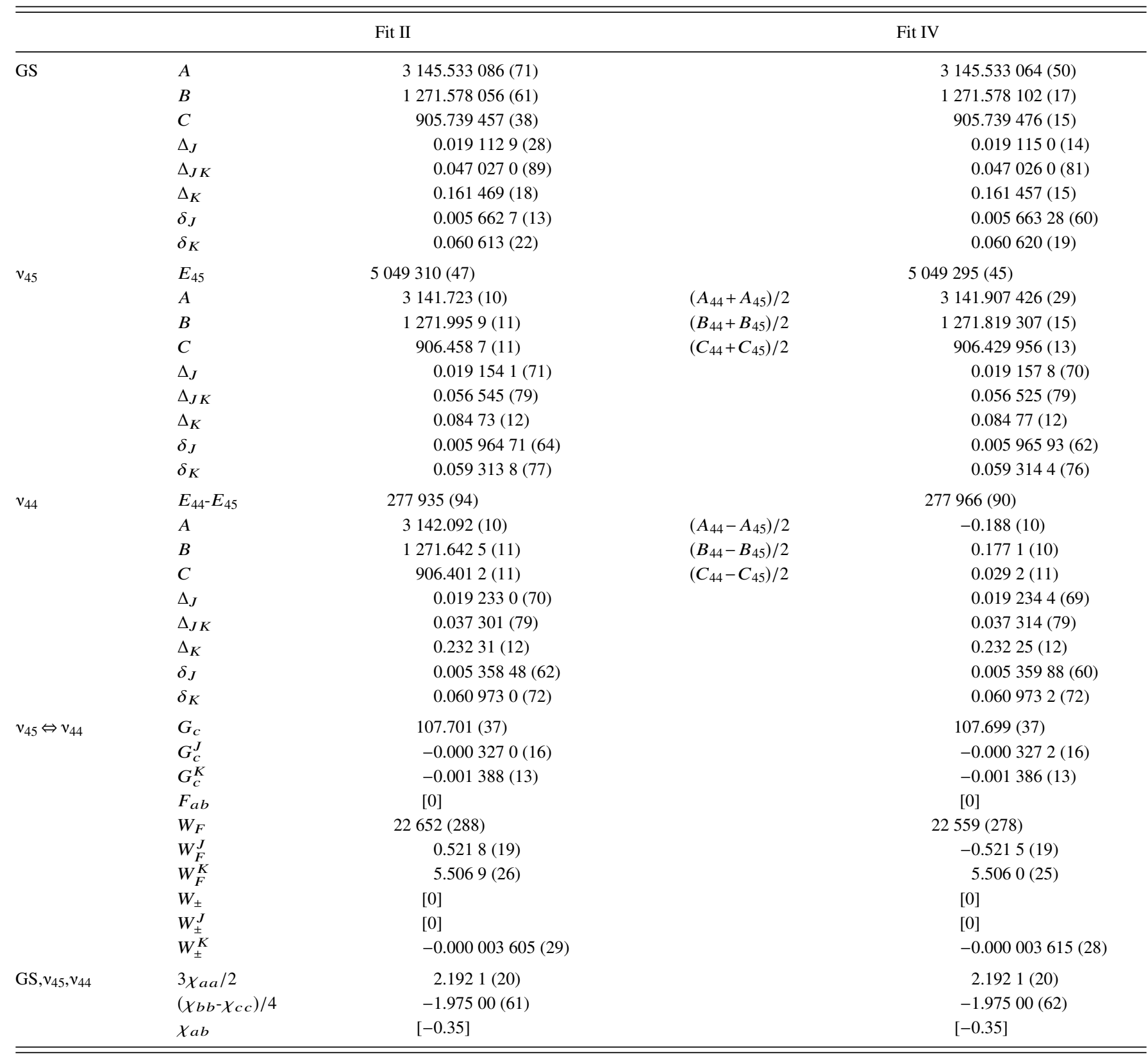

Despite the advantages of the theoretical approach discussed in Ref. 37, the accuracy of the absolute values of the IR frequencies, while sufficient for low resolution studies, is not yet suitable for a high resolution study involving perturbations for which a considerably higher accuracy is required.

In Table V, we show that the slightly negative GS and ES inertial defects are well reproduced by the calculations, as already discussed for doubly $\mathrm{N}$-substituted naphthalene derivatives. ${ }^{32}$ Noticeably, the ES inertial defect is overestimated for $v_{45}$ and slightly underestimated for $v_{44}$.

\section{Generalization to other PAHs and aza-derivatives}

To date, most of laboratory studies aiming at astronomical searches of PAH molecules have focused on their GS rotational spectra. In general, the lowest vibrational states lie at a relatively high energy (in comparison to the rotational pattern), avoiding perturbation of the GS rotational structure. In contrast

TABLE III. Stability of the effective vibrational frequencies resulting from the fitted parameters (in $\mathrm{MHz}$ unless indicated otherwise).

\begin{tabular}{lcr}
\hline \hline & $\begin{array}{c}\text { Hyperfine-free } \\
\text { (Fits I and III) }\end{array}$ & $\begin{array}{c}\text { Hyperfine } \\
\text { (Fits II and IV) }\end{array}$ \\
\hline$v_{45}$ & $5049310(47)$ & $5049294(45)$ \\
$v_{44}-v_{45}$ & $277935(94)$ & $277966(90)$ \\
$W_{F}$ & $22652(288)$ & $22559(278)$ \\
$E_{45}-E_{\mathrm{GS}}$ & $5047475.91(13)$ & $5047475.85(11)$ \\
$E_{44}-E_{45}$ & $281603.51(13)$ & $281603.52(13)$ \\
$E_{45}-E_{\mathrm{GS}} / \mathrm{cm}^{-1}$ & $168.365673(4)$ & $168.365671(4)$ \\
$E_{44}-E_{45} / \mathrm{cm}^{-1}$ & $9.393282(4)$ & $9.393282(4)$ \\
\hline \hline
\end{tabular}


TABLE IV. Differences between experimental and DFT values $(\Delta$ $=$ exp. - calc.) for the rotational constants and the state energy. The values are in $\mathrm{cm}^{-1}$

\begin{tabular}{lcccc}
\hline \hline & $\Delta A \times 10^{5}$ & $\Delta B \times 10^{5}$ & $\Delta C \times 10^{5}$ & $\Delta \mathrm{E}$ \\
\hline $\mathrm{GS}$ & -8.1 & 4.2 & 1.5 & \\
$v_{45}$ & -6.2 & 4.1 & 1.2 & 16.2 \\
$v_{44}$ & -10.3 & 4.0 & 1.3 & 15.9 \\
\hline \hline
\end{tabular}

TABLE V. Calculated and experimental values of the inertial defects in the GS, $v_{45}$, and $v_{44}$.

\begin{tabular}{lcc}
\hline & $\Delta_{\mathrm{i}}(\text { Calc. })^{\mathrm{a}}$ & \multicolumn{1}{c}{$\Delta_{\mathrm{i}}\left(\right.$ Expt. $^{\mathrm{a}}$} \\
\hline $\mathrm{GS}$ & -0.125 & $-0.13406(1)$ \\
$v_{45}$ & -0.706 & $-0.6411(9)$ \\
$v_{44}$ & -0.678 & $-0.6972(9)$ \\
\hline \hline
\end{tabular}

${ }^{\mathrm{a}}$ Inertial defect, $\Delta_{i}=I_{c}-I_{a}-I_{b}\left(\mathrm{u} \AA^{2}\right)$.

to the GS, it is clear from the relatively high density of states for such large molecules that Coriolis and Fermi interactions most probably occur in many IR bands of PAHs. Indeed, for symmetry reasons the pure rotational spectra within the lowest ES should be affected by Coriolis couplings since the two lowest states are often composed of a symmetric (butterfly-like) and asymmetric (ring puckering) out-of-plane deformations of the framework.

In the case of two-ring PAHs, for which a relatively high number of rotationally resolved data in the GS have been obtained, the two lowest modes are always out-of-plane deformations (butterfly and ring puckering) which possess very close energies. For example, the two lowest modes of naphthalene (for which no pure rotation spectrum can be observed in the GS and ES states due to symmetry) have $b_{3 u}$ (IR active) and $a_{u}$ (IR inactive) symmetries. No Fermi resonance is allowed but their direct product is $b_{3 g}$ (which contains the rotation about the $x$ axis); therefore, $c$-type Coriolis perturbation could be observed in the rotational energy levels of both states. The FT-FIR spectrum of naphthalene reported in Ref. 19 showed no clear perturbation in the $c$-type transitions, but we have noted in the present quinoline study that even for large perturbations of the rotational levels, the IR $c$-type transitions are not good tracers of such perturbations. For azulene, quinoxaline, and phthalazine, which belong to the $C_{2 \mathrm{v}}$ point group, pure rotational spectra of appreciable intensity result from their relatively high permanent dipole moment (often larger than 1 Debye). The two lowest states have $A_{2}$ and $B_{1}$ symmetries so that their direct product is $B_{2}$, which should also lead to Coriolis coupling about the $c$-axis. However, Christen et al. ${ }^{10}$ reported the study of the six lowest vibrational states of azulene at the microwave accuracy and did not report any perturbation in the rotational structures. It should be noted that their spectral range was limited to $20 \mathrm{GHz}$ so that only relatively low values of $J$ and $K_{a}$ were observed, which could explain why no perturbation was noticed. In the case of molecules belonging to the $C_{\mathrm{s}}$ point group such as most of the $\mathrm{N}$-bearing naphthalene derivatives (quinoline, isoquinoline, quinazoline, 1,6-naphthiridine), Fermi resonance together with $c$-type Coriolis coupling might occur in many cases between the two lowest states which are of $A^{\prime \prime}$ symmetry. In addition, the next stack of vibrational states is composed of five states and lies between $300 \mathrm{~cm}^{-1}$ and $400 \mathrm{~cm}^{-1}$, so it is obvious that the number of possible interacting states is rapidly increasing.

For larger PAHs, such as anthracene $\left(D_{2 h}\right)$, phenanthrene, acridine, and phenanthroline $\left(C_{2 \mathrm{v}}\right)$, the very same situation occurs as for naphthalene and azulene, respectively: By symmetry, no Fermi resonance is expected but Coriolis coupling about the $c$-axis is expected between the two lowest vibrational modes. For phenanthridine, which is the only three ring PANH belonging to the $C_{\mathrm{s}}$ point group with rotational information available in the GS, the calculated lowest vibrational modes have $A^{\prime \prime}$ symmetries and relatively close energies, most probably leading to both Fermi and Coriolis perturbations in the ES rotational structures.

Therefore, for relatively large PAHs and their derivatives, most of the ES rotational structures should be affected by many perturbations and their accurate prediction will require thorough laboratory investigations with complementary techniques.

\section{CONCLUSION}

In this paper, we report the results of a thorough spectroscopic study of the rotational structure of the three lowest vibrational states of quinoline (GS, $v_{45}$, and $\left.v_{44}\right)$. While the GS rotational structure is unperturbed and very accurately simulated using purely rotational terms of the Watson $A$-reduction Hamiltonian, the rotational patterns belonging to the $v_{45}$ and $v_{44}$ ES show relatively large perturbations requiring specialized treatment. Considerable $\Delta K_{a}=2$ perturbations were observed for all values of $K_{a}$ that were extensively sampled in the range of our observations and a further $\Delta K_{a}=2$ perturbation at higher $K_{a}$ values was also identified and measured. In order to thoroughly understand such perturbations in the rotational patterns of the ES, we used complementary high resolution spectroscopic techniques to obtain the most accurate set of molecular constants possible. The complete dataset was simultaneously fitted to the experimental accuracy using the Watson A-reduction Hamiltonian augmented with Coriolis and Fermi vibration-rotation coupling terms and hyperfine nuclear spin-quadrupole coupling parameters for the nitrogen nucleus. Accurate values of the rotational constants were derived by fitting linear combinations of $A, B, C$ instead of a straightforward fit of each of them independently. Accurate values of the vibrational energies were obtained by reducing the three strongly correlated parameters of fit (two energies and the Fermi term) to two effective vibrational separations with correlations determined by error propagation making use also of the off-diagonal elements of the variance covariance matrix (see Eq. (6) of Ref. 55).

We compared our experimentally derived parameters for the three states to those from anharmonic DFT calculations. As already shown in a previous paper, the calculated rotational constants obtained using this method/basis set combination are in very good agreement with the experimental ones. These reliable predictions turned out to be very useful for setting initial conditions for the challenging rotational analysis of such complicated spectra. Experimental and calculated inertial 
defects in the GS and ES also agree well, which is another validation of the calculations in view of the high sensitivity of this parameter to deviations. On the other hand, the accuracies of the absolute vibrational frequencies and, to a larger extent, of the energy difference between vibrational modes remain insufficient for a high resolution study, which hampers the usefulness of the calculated values for setting initial conditions for fitting interaction parameters.

The perturbations observed here are expected to be ubiquitous in the lowest vibrational states of PAHs and their derivatives. Even for the two lowest ES of two rings PAHs (smallest species of this family), the rotational structures in the ES might often be affected by Coriolis perturbations. Increasing the size of the molecule (i.e., the density of vibrational states) will lead to an increase in the number of the possible interacting vibrational states. This has to be accounted for when recording pure rotational spectra of such species in order to be able to generate reliable linelists for radioastronomical searches.

\section{ACKNOWLEDGMENTS}

The authors are grateful to SOLEIL and the AILES beamline staff for providing synchrotron beam under the proposal 20131274, as well as to the French ANR agency for supporting this work through Contract No. ANR-10-BLAN050. Z.K. acknowledges a grant from the Polish National Science Centre (Decision No. DEC/2011/02A/ST2/00298).

\footnotetext{
${ }^{1}$ A. Léger and J. L. Puget, Astron. Astrophys. 137, L5 (1984).

${ }^{2}$ L. J. Allamandola, A. G. G. M. Tielens, and J. R. Barker, Astrophys. J. 290, L25-L28 (1985).

${ }^{3}$ A. G. G. M. Tielens, Annu. Rev. Astron. Astrophys. 46, 289 (2008).

${ }^{4}$ E. Herbst and E. F. van Dishoeck, Annu. Rev. Astron. Astrophys. 47, 427 (2009).

${ }^{5}$ J. Cernicharo, A. M. Heras, A. G. G. M. Tielens, J. R. Pardo, F. Herpin, M. Guélin, and L. B. F. M. Waters, Astrophys. J., Lett. 546, L123 (2001).

${ }^{6}$ S. B. Charnley, Y. J. Kuan, H. C. Huang, O. Botta, H. M. Butner, N. Cox, D. Despois, P. Ehrenfreund, Z. Kisiel, Y. Y. Lee, A. J. Markwick, Z. Peeters, and S. D. Rodgers, Adv. Space Res. 36, 137-145 (2005).

${ }^{7}$ P. Pilleri, D. Herberth, T. F. Giesen, M. Gerin, C. Joblin, G. Mulas, G. Malloci, J. U. Grabow, S. Brunken, L. Surin, B. D. Steinberg, K. R. Curtis, and L. T. Scott, Mon. Not. R. Astron. Soc. 397, 1053-1060 (2009).

${ }^{8}$ S. Kwok and Y. Zhang, Astrophys. J. 771, 5 (2013).

${ }^{9}$ Y. Ali-Haïmoud, Mon. Not. R. Astron. Soc. 437, $2728-2743$ (2014).

${ }^{10}$ P. Christen, A. Bauder, and Hs. H. Günthard, J. Mol. spectrosc. 43, 1 (1972).

${ }^{11}$ Z. Kisiel, O. Desyatnyk, L. Pszczolkowski, S. B. Charnley, and P. Ehrenfreund, J. Mol. Spectrosc. 217, 115 (2003).

${ }^{12}$ F. J. Lovas, R. J. McMahon, J.-U. Grabow, M. Schnell, J. Mack, L. T. Scott, and R. L. Kuczkowski, J. Am. Chem. Soc. 127, 4345 (2005).

${ }^{13}$ S. Thorwirth, P. Theule, C. A. Gottlieb, M. C. McCarthy, and P. Thaddeus, Astrophys. J. 662, 1309 (2007).

${ }^{14}$ D. McNaughton, P. D. Godfrey, R. D. Brown, S. Thorwirth, and J.-U. Grabow, Astrophys. J. 678, 309 (2008).

${ }^{15}$ D. McNaughton, P. D. Godfrey, M. K. Jahn, D. A. Dewald, and J.-U. Grabow, J. Chem. Phys. 134, 154305 (2011).

${ }^{16}$ S. Albert, K. K. Albert, P. Lerch, and M. Quack, Faraday Discuss. 150, 71 (2011).

${ }^{17}$ B. E. Brumfield, J. T. Stewart, and B. J. McCall, J. Phys. Chem. Lett. 3, 1985 (2012).

${ }^{18}$ O. Pirali, V. Boudon, J. Oomens, and M. Vervloet, J. Chem. Phys. 136, 024310 (2012).
}

${ }^{19}$ O. Pirali, M. Goubet, T. R. Huet, R. Georges, P. Soulard, P. Asselin, J. Courbe, P. Roy, and M. Vervloet, Phys. Chem. Chem. Phys. 15, 10141 (2013).

${ }^{20}$ G. Malloci, C. Joblin, and G. Mulas, Chem. Phys. 332, 353-359 (2007).

${ }^{21}$ M. Basire, P. Parneix, and F. Calvo, J. Chem. Phys. 129, 081101 (2008).

${ }^{22}$ B. J. Esselman, B. K. Amberger, D. Shutter, A. Mitchell, J. F. Stanton, R. C. Woods, and R. J. McMahon, J. Chem. Phys. 139, 224304 (2013).

${ }^{23}$ S. C. Wait and J. C. McNerney, J. Mol. Spectrosc. 34, 56 (1970).

${ }^{24}$ M. A. Martin-Drumel, O. Pirali, Y. Loquais, C. Falvo, and P. Bréchignac, Chem. Phys. Lett. 557, 53 (2013).

${ }^{25}$ O. Pirali, V. Boudon, N. Carrasco, and E. Dartois, Astron. Astrophys. 561, A109 (2014).

${ }^{26}$ J.-B. Brubach, L. Manceron, M. Rouzieres, O. Pirali, D. Balcon, F. K. Tchana, V. Boudon, M. Tudorie, T. R. Huet, A. Cuisset et al., AIP Conf. Proc. 1214, 81 (2010).

${ }^{27}$ F. Matsushima, H. Odashima, T. Iwasaki, S. Tsunekawa, and K. Takagi, J. Mol. Struct. 352, 371 (1995); V. M. Horneman, R. Anttila, S. Alanko, and J. Pietila, J. Mol. Spectrosc. 234, 238 (2005).

${ }^{28}$ G. Mouret, M. Guinet, A. Cuisset, L. Croize, S. Eliet, R. Bocquet, and F. Hindle, IEEE Sens. J. 13, 133 (2013).

${ }^{29}$ I. Medvedev, M. Winnewisser, F. C. De Lucia, E. Herbst, E. BialkowskaJaworska, L. Pszczolkowski, and Z. Kisiel, J. Mol. Spectrosc. 228, 314 (2004).

${ }^{30}$ Z. Kisiel and A. Krasnicki, J. Mol. Spectrosc. 262, 82 (2010).

${ }^{31}$ S. Kassi, D. Petitprez, and G. Wlodarczak, J. Mol. Struct. 517-518, 375 (2000).

${ }^{32}$ S. Gruet, M. Goubet, and O. Pirali, J. Chem. Phys. 140, 234308 (2014).

${ }^{33}$ S. Kassi, D. Petitprez, and G. Wlodarczak, J. Mol. Spectrosc. 228, 293 (2004).

${ }^{34}$ M. Krüger and H. Dreizler, Z. Naturforsch. A 45, 724 (1990).

${ }^{35}$ M. Krüger, H. Harder, C. Gerke, and H. Dreizler, Z. Naturforsch. A 48, 737 (1993).

${ }^{36}$ M. J. Frisch, G. W. Trucks, H. B. Schlegel, G. E. Scuseria, M. A. Robb, J. R. Cheeseman, G. Scalmani, V. Barone, B. Mennucci, G. A. Petersson, H. Nakatsuji, M. Caricato, X. Li, H. P. Hratchian, A. F. Izmaylov, J. Bloino, G. Zheng, J. L. Sonnenberg, M. Hada, M. Ehara, K. Toyota, R. Fukuda, J. Hasegawa, M. Ishida, T. Nakajima, Y. Honda, O. Kitao, H. Nakai, T. Vreven, J. A. Montgomery, Jr., J. E. Peralta, F. Ogliaro, M. Bearpark, J. J. Heyd, E. Brothers, K. N. Kudin, V. N. Staroverov, R. Kobayashi, J. Normand, K. Raghavachari, A. Rendell, J. C. Burant, S. S. Iyengar, J. Tomasi, M. Cossi, N. Rega, J. M. Millam, M. Klene, J. E. Knox, J. B. Cross, V. Bakken, C. Adamo, J. Jaramillo, R. Gomperts, R. E. Stratmann, O. Yazyev, A. J. Austin, R. Cammi, C. Pomelli, J. W. Ochterski, R. L. Martin, K. Morokuma, V. G. Zakrzewski, G. A. Voth, P. Salvador, J. J. Dannenberg, S. Dapprich, A. D. Daniels, Ö. Farkas, J. B. Foresman, J. V. Ortiz, J. Cioslowski, and D. J. Fox, GAUSSIAN 09, Revision B.01 (Gaussian, Inc., Wallingford CT, 2009).

${ }^{37}$ M. Goubet and O. Pirali, J. Chem. Phys. 140, 044322 (2014).

${ }^{38}$ W. Lodyga, M. Kreglewski, P. Pracna, and S. Urban, J. Mol.Spectrosc. 243, 182 (2007).

${ }^{39}$ Z. Kisiel, L. Pszczolkowski, I. R. Medvedev, M. Winnewisser, F. C. De Lucia, and E. Herbst, J. Mol. Spectrosc. 223, 231-243 (2005).

${ }^{40}$ Z. Kisiel, L. Pszczolkowski, B. J. Drouin, C. S. Brauer, S. Yu, J. C. Pearson, I. R. Medvedev, S. Fortman, and C. Neese, J. Mol. Spectrosc. 280, 134 (2012).

${ }^{41}$ H. M. Pickett, J. Mol. Spectrosc. 148, 371 (1991), see http://spec.jpl.nasa. gov.10.1016/0022-2852(91)90393-O.

${ }^{42}$ C. M. Western, Pgopher, A program for simulating rotational structure (University of Bristol, 2014), see http://pgopher.chm.bris.ac.uk.

${ }^{43}$ Z. Kisiel, E. Bialkowska-Jaworska, and L. Pszczolkowski, J. Mol. Spectrosc. 177, 240 (1996).

${ }^{44}$ Z. Kisiel and L. Pszczolkowski, J. Mol. Spectrosc. 178, 125 (1996).

${ }^{45}$ Z. Kisiel, PROSPE - Programs for Rotational Spectroscopy (Institute of Physics, Polish Academy of Sciences, 2000), see http://info.ifpan.edu.pl/ kisiel/prospe.htm.

${ }^{46} \mathrm{Z}$. Kisiel, Spectroscopy from Space, edited by J. Demaison, K. Sarka, and E. A. Cohen (Kluwer Academic Publishers, Dordrecht, 2001), pp. 91-106.

${ }^{47}$ Z. Kisiel, O. Dorosh, M. Winnewisser, M. Behnke, I. R. Medvedev, and F. C. De Lucia, J. Mol. Spectrosc. 246, 39 (2007).

${ }^{48}$ R. A. H. Butler, D. T. Petkie, P. Helminger, F. C. De Lucia, E. B. Jaworska, and Z. Kisiel, J. Mol. Spectrosc. 244, 113 (2007).

${ }^{49}$ Bechtel et al., Angew. Chem., Int. Ed. 47, 2969 (2008).

${ }^{50}$ J. Kraitchman and B. P. Dailey, J. Chem. Phys. 22, 1477 (1954).

${ }^{51}$ M. E. Sanz, M. C. McCarthy, and P. Thaddeus, J. Chem. Phys. 119, 11715 (2003).

52 J. K. G. Watson, Vibrational Spectra and Structure, edited by J. R. Durig (Elsevier, New York/Amsterdam, 1977), Vol. 6, pp. 1-89. 
${ }^{53}$ D. Papoušek and M. R. Aliev, Molecular Vibrational/Rotational spectra (Academia, Prague, 2001).

${ }^{54}$ A. Krasnicki, Z. Kisiel, W. Jabs, B. P. Winnewisser, and M. Winnewisser, J. Mol. Spectrosc. 267, 144-149 (2011).

${ }^{55}$ Z. Kisiel, E. B. Jaworska, R. A. H. Butler, D. T. Petkie, P. Helminger, I. R. Medvedev, and F. C. De Lucia, J. Mol. Spectrosc. 254, 78 (2009).
${ }^{56}$ See supplementary material at http://dx.doi.org/10.1063/1.4913750 for Results of Fit II and Fit IV (files FitII.txt and FitIV.txt). Calculated Cartesian coordinates in principal axis orientation of quinoline are given in file CartesianCoodinates.doc. Calculated harmonic and anharmonic frequencies and rotational constants of the vibrational states of quinoline are given in the file VibRot.doc. 